\section{O Aprendizado sobre a Hanseníase e Tuberculose a partir de um Projeto de Cultura e Extensão: Relato de Experiência}

\author{
Learning about Leprosy and Tuberculosis from a Project of \\ Culture and Extension: an Experience Report
}

\section{RESUMO}

O presente trabalho tem por objetivo relatar a experiência de bolsistas no desenvolvimento de atividades relacionadas ao projeto de cultura e extensão Hanseníase e Tuberculose na Atenção Primária: organização de dados e busca ativa em uma unidade básica de saúde no período de 2010 a 2014. Estudo qualitativo, descritivo que tem por material empírico a reflexão dos bolsistas. Como potencialidades aponta-se a sensibilização da equipe da Unidade Básica de Saúde (UBS), em especial, dos agentes comunitários de saúde com relação ao tema da hanseníase e tuberculose; aumento da busca ativa de casos; realização de atividades em sala de espera; articulação do nível distrital e a equipe da UBS com relação à responsabilização pelos casos; vivência dos estudantes da graduação em visitas domiciliares, atendimento individual e atividades educativas às famílias com hanseníase e tuberculose. A partir da vivência houve a fundação da Liga de Hanseníase e articulação ao Movimento de Reintegração das Pessoas Atingidas pela Hanseníase. Como limites da experiência considera-se a não realização das atividades desenvolvidas pelos bolsistas pela equipe da UBS e a participação de estudantes de outros cursos da área de saúde. O projeto promoveu aprendizados na organização de atividades coletivas, liderança e a produção de pesquisas.

Palavras-chave: Hanseníase. Tuberculose. Atenção Primária à Saúde.

\section{ABSTRACT}

This work has the objective to report the experience of scholarship students in the development of activities related to the project of culture and extension Leprosy and Tuberculosis in Primary Attention: organization of databases and active search in an basic health unit in a period of 2010 to 2014. Qualitative and descriptive study whose empirical material is the reflection made by scholarship students. As potentiality points the sensibility of the Basic Health Unit (BHU) team, in especial by the community

\section{Marcela Gonçalves, \\ KAREN da Silva \\ SANTOS, \\ Fabiana Ribeiro \\ Santana e Cinira \\ Magali Fortuna \\ Universidade de São Paulo. Escola de Enfermagem de Ribeirão Preto, São Paulo, Brasil}


health agents in the issue of leprosy and tuberculosis; the increase in the active search of cases; waiting room activities implementation; articulation of district level and the BHU team in the concern of responsibility by the cases; undergraduate students' experiences in home visits, individual care and educative activities to the families with leprosy and tuberculosis. From the experiences, there was the foundation of the Leprosy League in articulation with the Reintegration Movement of People Affected by Leprosy. As the limit of experiments, the non realization of the activities developed by scholarship students by the BHU team and the participation of students from other health courses are considered. The project promotes the learning in the organization of collective activities, leadership and research production.

Keywords: Leprosy. Tuberculosis. Primary Health Care.

\section{INTRODUÇÃO}

No Brasil e em outros países, mais de um bilhão de pessoas vivem com menos de US\$2 por dia. Essa população sofre com todo tipo de carência, como água potável, escolaridade, saneamento básico, moradia, acesso aos serviços de saúde e também são vítimas de doenças negligenciadas [1]. Entre as doenças negligenciadas no Brasil estão à hanseníase e a tuberculose [2].

A hanseníase é uma doença crônica, infecciosa, de grande importância para a saúde pública, por seu alto poder incapacitante. Prejudica os nervos periféricos, mas também, pode acometer articulações, olhos, testículos, gânglios e outros órgãos. Seu grau incapacitante está ligado à capacidade de penetração do bacilo Mycobacterium leprae na célula nervosa [3].

A hanseníase é considerada de baixa transmissibilidade. A transmissão é através da via respiratória, sendo os pacientes multibacilares (que apresentam baciloscopia positiva e/ou que apresentam mais de cinco lesões cutâneas) a principal fonte de infecção [3].

Em relação aos dados epidemiológicos da hanseníase, a Ásia apresentou a maior taxa de detecção, 9,39 casos por 100.000 habitantes, seguida das Américas com 4,58 casos por 100.000 habitantes. Nessas regiões os dados foram fortemente influenciados pelo número de casos notificados pela Índia com 133.717, maior número de casos, e pelo Brasil com 37.610 casos, o segundo país em número de casos. Dos 40.474 casos novos nas Américas 93\% são casos notificados no Brasil [10].

A tuberculose também é uma doença infecciosa, causada pelo Mycobacterium tuberculosis. É transmitida de pessoa a pessoa, principalmente, através do ar e em ambientes fechados [3].

Uma vez em contato com o bacilo, a pessoa poderá desenvolver a tuberculose em qualquer fase da sua vida. Isso ocorre porque o sistema imunológico não consegue mais manter os bacilos sob controle. Os órgãos mais comumente afetados são: pulmões, glânglios linfáticos, pleura, laringe, rins, cérebro e ossos [3].

Em relação aos casos de tuberculose, o Brasil é um dos 22 países priorizados pela Organização Mundial de Saúde (OMS) que concentram 80\% da carga mundial. Em 
2009, foram notificados 72 mil casos novos, correspondendo a um coeficiente de incidência de 38/100.00o habitantes. Destes, 41 mil foram bacilíferos. Esses indicadores colocam o Brasil na $19^{\mathrm{a}}$ posição em relação ao número de casos e na $104^{\mathrm{a}}$ posição em relação ao coeficiente de incidência [9].

Em Ribeirão Preto, o seguimento dessas doenças está centralizado nas Unidades Distritais de Saúde, o que pode ocasionar dificuldades para as unidades básicas de saúde (UBS) em envolver-se com ações de busca ativa, controle e acompanhamento dos casos, uma vez que a centralização pode dificultar acesso dos pacientes e desresponsabilizar os profissionais da atenção básica quanto aos cuidados para com as pessoas acometidas e mesmo para com a prevenção e controle das mesmas.

Para a melhoria do cuidado faz-se necessário o envolvimento cotidiano da equipe na busca ativa de casos dessas doenças com ações coordenadas e ininterruptas, assim como a integração entre as instituições formadoras e os serviços de saúde.

O vínculo entre ensino e serviço de saúde contribui para a elaboração e aperfeiçoamento de atividades desenvolvidas nos serviços de saúde, promovendo melhoria das informações coletadas e também dos bancos de dados do Sistema Único de Saúde (SUS). Desse modo, os projetos de extensão devem ser incentivados e valorizados, pois criam parcerias entre profissionais das universidades e dos serviços de saúde, propiciando um ambiente de discussão para que os problemas da prática cotidiana possam ser incluídos às atividades de pesquisa e também sensibilizar os graduandos [4].

Nesse sentido, a Escola de Enfermagem de Ribeirão Preto da Universidade de São Paulo (EERP-USP), desenvolveu um projeto de extensão universitária Hanseníase e Tuberculose na Atenção Primária: organização de dados e busca ativa em uma unidade básica de saúde no período de 2010 a 2014, envolvendo estudantes da graduação.

Esse projeto teve por objetivo manter viva, na pauta da equipe de saúde, a atenção para com as medidas de prevenção e controle em relação à hanseníase e a tuberculose. E ainda propor, realizar e avaliar, em conjunto com a equipe local da UBS da Vila Recreio, localizada no município de Ribeirão Preto-SP, ações de busca ativa de tuberculose e hanseníase; visitas domiciliares para pacientes que estão iniciando o tratamento e em seguimento, para assegurar a continuidade no tratamento; identificar e sistematizar a relação dos casos de tuberculose e hanseníase da área de abrangência da UBS da Vila Recreio.

Outro projeto de extensão universitária que visa o combate à hanseníase é a Liga de Hanseníase Profa. Dra. Maria Helena Pessini de Oliveira, uma entidade civil com sede na EERP-USP. A Liga permite aos alunos de graduação, pós-graduação e trabalhadores da área da saúde aprofundar o conhecimento teórico e prático na área de hansenologia, bem como busca promover a discussão de assuntos relacionados à hanseníase no âmbito individual, coletivo e da gestão na área de saúde [7].

A Liga trabalha integrada com o Movimento de Reintegração das Pessoas Atingidas pela Hanseníase (Morhan) que é uma entidade sem fins lucrativos, que promove atividades para eliminação da Hanseníase, através de conscientização e construção de políticas públicas [5].

Procuramos relatar nesse trabalho as seguintes questões: Como tem se dado esses trabalhos de extensão universitária? Quais são as suas potencialidades e os limites sob 
a ótica de quem tem vivido como estudante bolsista?

\section{OBJETIVO}

Relatar a experiência de bolsistas no desenvolvimento de atividades relacionadas ao projeto de cultura e extensão: Hanseníase e Tuberculose na Atenção Primária: organização de dados e busca ativa em uma unidade básica de saúde.

\section{METODOLOGIA}

Trata-se de um relato de experiência vivenciado no Projeto de Cultura e Extensão no período de agosto de 2010 a junho de 2014.

Este projeto ainda vem se desenvolvendo por graduandos da EERP-USP com apoio do Programa Aprender com Cultura e Extensão.

A população atingida pelo projeto foi: usuários em tratamento, alta e/ou abandono de tratamento de tuberculose e/ou hanseníase, comunicantes, sintomáticos respiratórios ou dermato-neurológicos pertencentes a área de abrangência da UBS Vila Recreio. Também foram envolvidos profissionais da UBS e usuários em geral que participaram de ações informativas como grupos em sala de espera.

As estratégias desenvolvidas pelos bolsistas em parceria com o ensino e o serviço de saúde foram:

" Sistematização e atualização dos dados, em que o aluno estabelecia o vínculo entre o centro de referência e a UBS trazendo informações sobre os casos novos e em tratamento de hanseníase e tuberculose;

" Realização de visitas domiciliares com os agentes comunitários de saúde (ACS) e equipe de enfermagem aos casos em tratamento, abandono ou cura;

" Discussão de casos com a equipe e orientações em relação às enfermidades;

" Orientações em sala de espera da UBS;

" Rodas de conversa com representantes religiosos do bairro da UBS;

" Discussões na sede das entidades religiosas sobre hanseníase e tuberculose;

" Atividades educativas com alunos do ensino médio de uma escola do bairro onde a UBS está vinculada e uma atividade educativa com os ACS em parceria com Morhan e a Liga de Hanseníase Professora Maria Helena Pessini de Oliveira da EERP-USP;

"Contato com o Centro Saúde-Escola do Sumarezinho ao menos uma vez por mês para atualização dos usuários em tratamento de tuberculose e hanseníase da área de abrangência da UBS Vila Recreio. Para tal é organizada uma planilha mensal com nome, endereço, número Hygia*, data do início do seguimento e atual con-

*O município de Ribeirão Preto utiliza-se do Hygia, sistema de gestão da saúde que efetua a Gestão da Saúde Pública em Municípios e Estados. O sistema interliga todas as unidades da rede de atendimento pessoal [6]. 
dição do tratamento;

" Ações de busca ativa e visitas domiciliares junto com os profissionais da UBS, como ACS e enfermeiros, e estudantes da EERP-USP que estiveram em atividades curriculares da disciplina de Cuidado Integral a Saúde II.

\section{RESULTADOS E DISCUSSÃO}

Durante o processo de implementação das ações de saúde pelos bolsistas em parceria com o ensino e o serviço de saúde observou-se uma maior sensibilização por parte da equipe da UBS Vila Recreio no acompanhamento dos casos de tuberculose e hanseníase da área de abrangência.

O vínculo e olhar atento das equipes de saúde (UBS e distrito) auxiliam a adesão ao tratamento, pois o paciente sente-se apoiado e acompanhado. Desta maneira, vê-se a importância de equipes com um olhar mais sensibilizado para essas duas doenças e que garanta a continuidade do paciente no tratamento.

Essa sensibilização traduz-se pelo aumento de exames físicos e vacinação com a BCG (Bacillus Calmette-Guérin) para os comunicantes, incentivo à coleta de escarro para sintomáticos respiratórios, solicitação de visitas domiciliares em conjunto com o docente da EERP-USP e estudantes, questionamentos e interesse em saber sobre outras formas de tuberculose e seu tratamento.

O exame físico para detecção de sinais e sintomas de hanseníase inclui: exame físico geral; exame dermatoneurológico (exame da superfície corporal, teste de sensibilidade nas lesões suspeitas); avaliação neurológica simplificada (palpação de nervos); teste de força muscular; teste de sensibilidade de córnea, palmas e plantas o que determina o grau de incapacidade nas mãos, pés, olhos [3]. E a vacinação com a BCG é indicada para os contatos intradomiciliares de pacientes diagnosticados com hanseníase [2].

A realização de visitas domiciliares é fundamental para o conhecimento do contexto social do portador da doença e de seus familiares e propicia a criação de vínculos. Nessa direção, conhecer melhor o ambiente no qual os pacientes estão inseridos facilita no manejo de intercorrências durante o tratamento e possibilita novas formas de interação com o indivíduo e a família [8]. Além disso, a visita domiciliar aos portadores da hanseníase e tuberculose busca desenvolver uma maior sensibilização dos estudantes, contribuindo para a formação do olhar crítico-reflexivo do futuro enfermeiro.

Os ACS também contribuem na busca ativa para a detecção dos casos suspeitos. Durante a visita domiciliar são identificadas as pessoas que convivem com o doente, sendo encaminhadas ao distrito para avaliação.

Segundo os próprios ACS, os mesmos estão mais sensibilizados e com informações mais precisas sobre essas duas patologias. Isso aumenta consideravelmente a possibilidade de que haja encaminhamento precoce das pessoas com tosse persistente para coleta de escarro e de pessoas com manchas ou perda de sensibilidade para avaliação na unidade de saúde.

Estimamos que durante o período de desenvolvimento do projeto mais de $50 \mathrm{fa}$ mílias receberam visita domiciliar. Os casos suspeitos foram informados à enfermeira 
da Unidade para encaminhamento ao Centro de Referência. Cerca de dez coletas de escarros foram realizadas em domicílio. Todos os usuários em tratamento de hanseníase e tuberculose da área de abrangência da UBS da Vila Recreio foram visitados. Essas ações contribuíram para adesão dos pacientes ao tratamento, remissão de dúvidas e apoio aos mesmos e suas famílias. Não houve sistematização quanto ao número de pacientes diagnosticados a partir das ações desenvolvidas.

Um desdobramento do projeto de extensão foi a fundação da Liga de Hanseníase em parceria com o Morhan (Movimento de Reintegração das pessoas Atingidas pela Hanseníase), que originou-se a partir da vivência da primeira bolsista em uma atividade no distrito de saúde com os usuários e familiares de pessoas com hanseníase. Tal vivência a levou a conhecer o Morhan e, Karen da Silva Santos, pós-graduanda militante nacional do movimento, sensibilizou-a para iniciar a organização da Liga de Hanseníase.

A Liga de Hanseníase e o Morhan proporcionam ao graduando um ambiente de comunicação e aprendizado. As reuniões da Liga e do Morhan são quinzenais fazendo com que o tema da hanseníase esteja constantemente em pauta no campus da USP de Ribeirão Preto.

Com a Liga de Hanseníase houve a aproximação com a coordenação municipal do programa de hanseníase que culminou em atividades no Dia Mundial de Combate a Hanseníase (último domingo do mês de janeiro) e na participação da Campanha dos 3 bichos: Geo-helmintíase, Tracoma e Hanseníase no segundo semestre de 2013 e no primeiro semestre de 2014 .

O aprendizado é importante não somente para o bolsista envolvido no projeto de cultura e extensão, mas também para os estudantes vinculados à disciplina Cuidado Integral em Saúde II, formando profissionais com a capacidade de prestar um cuidado qualificado com um olhar mais atento para essas duas patologias, uma vez que a busca e atualização se faz constantemente.

O futuro enfermeiro também desenvolve a criatividade, pois o projeto de cultura e extensão exige diversos métodos e estratégias para realização das atividades junto à comunidade e equipe.

A partir da convivência com outros profissionais, o aluno desenvolve a capacidade de liderança e também aprende a conviver em grupo, planejando e avaliando intervenções.

Concomitante a isso, um resultado indireto do projeto é a formação de futuros enfermeiros sensibilizados para a identificação e o seguimento de pessoas e comunicantes de hanseníase e tuberculose.

O projeto de extensão também contribui para a experiência dos estudantes com elaboração de pôsteres incentivando a equipe local a refletir sobre o trabalho realizado e a apresentar em eventos. É o caso do Expo Saúde, realizado anualmente pela Secretaria Municipal da Saúde de Ribeirão Preto e que tem por objetivo incentivar as equipes de saúde a apresentarem suas experiências exitosas em direção à qualificação da atenção prestada.

Para a pesquisa, as contribuições do projeto de extensão estão relacionadas à elaboração e desenvolvimento de duas iniciações científicas, cujo títulos são: Significados e Sentidos da Hanseníase para as pessoas que viveram na era da poliquimioterapia e 
na era sulfônica e Significados e Sentidos da Hanseníase para os trabalhadores da Saúde.

Um dos limites do trabalho é o de que ainda há a necessidade de se ter um bolsista para fazer a integração entre o Centro Saúde-Escola Sumarezinho e a UBS, pois as equipes referem não ter tempo para essa articulação. Quando o bolsista não está presente nota-se que a equipe tende a voltar ao seu modo habitual de funcionamento delegando ao ambulatório especializado (no distrito) o acompanhamento. Desta forma, não se mantém um segmento atento durante o tratamento, com os comunicantes, além de educação da população para com os cuidados das doenças.

É um limite do trabalho a não sistematização exata do numero de casos diagnosticados pelo trabalho efetuado pelos estudantes o que está em vias de revisão para os próximos anos.

O envolvimento de estudantes de outros cursos no projeto é um fator limitante, como estudantes de medicina, psicologia, fisioterapia, entre outros, pois a unidade recebe majoritariamente estudantes do curso de Enfermagem. Considera-se que a formação de futuros profissionais para busca ativa, diagnóstico precoce e tratamento adequado ainda desafia e projetos dessa natureza podem favorecer esse aspecto e ainda impactar no trabalho em equipe.

A Liga de Hanseníase é composta principalmente por graduandos de Enfermagem e possui apenas um dentista que é pós-graduando atualmente. Um dos objetivos para os próximos semestres é sensibilizar outros estudantes.

\section{CONCLUSÃO}

O Projeto de Cultura e Extensão colocou na agenda dos trabalhadores da UBS da Vila Recreio os temas da hanseníase e tuberculose. Também permitiu atualização dos ACS e maior integração entre o nível local e distrital com relação aos casos residentes na área de abrangência.

Houve a criação de uma Liga de Hanseníase e o envolvimento de estudantes durante a graduação relacionada às atividades comunitárias de combate à hanseníase e à tuberculose.

Destaca-se que sem esse projeto os estudantes da graduação poderiam não ter a oportunidade de envolverem-se com os agravos ou envolverem-se pouco e após a formação não atentarem-se a esses tipos de agravos, ocasionando num diagnóstico tardio ou equivocado.

Outro fator importante foi a sensibilização da comunidade para as questões de estigma e preconceito que cercam tanto a tuberculose quanto a hanseníase desde os tempos mais remotos.

Assim destaca-se a importância do projeto para os participantes bolsistas com vivencias de liderança e de trabalho em equipe para organizar as ações na UBS, no distrito sanitário, no município, junto ao Morhan e a Liga de Hanseníase. 
[1] ASSAD, L. Doenças negligenciadas estão nos países pobres e em desenvolvimento. Cienc. Cult., São Paulo, v. 62, n. 1, 2010.

[2] BRASIL. Ministério da Saúde. Departamento de Ciência e Tecnologia, Secretaria de Ciência, Tecnologia e Insumos Estratégicos. Neglected diseases: the strategies of the Brazilian Ministry of Health. Rev. Saúde Pública, v. 44, n. 1, p. 200-2, 2010. DOI: http://dx.doi.org/10.1590/So034-89102010000100023.

[3] BRASIL. Ministério da Saúde. Secretaria de Atenção à Saúde. Departamento de Atenção Básica. Cadernos de Atenção Básica. Vigilância em Saúde: dengue, esquistossomose, hanseníase, malária, tracoma e tuberculose. Brasília: Ministério da Saúde, 2008.

[4] MATHIAS, T. A. de F. et al. Atividades de extensão universitária em comitê de prevenção de mortalidade infantil e estatísticas de saúde. Rev. Bras. Enferm., v. 62, n. 2, p. 205-311, 2009. DOI: http://dx.doi.org/10.1590/ So034-71672009000200022.

[5] MOVIMENTO DE REINTEGRAÇÃO DAS PESSOAS ATINGIDAS PELA HANSENÍASE (MORHAN). Institucional. 2014. Disponível em: <http:// www.morhan.org.br/institucional>. Acesso em: 8 jul. 2014.

[6] PREFEITURA DE RIBEIRÃO PRETO. Sistema Hygia. 2014. Disponível em: <http://www.techne.com.br/casos/CaseHygia_RibeiraoPreto.pdf >. Acesso em: 8 jul. 2014.

[7] UNIVERSIDADE DE SÃO PAULO. Escola de Enfermagem de Ribeirão Preto. Liga de Hanseníase Profa. Dra. Maria Helena Pessini de Oliveira. Estatuto, Regimentos e Regulamentos. Ribeirão Preto: Universidade de São Paulo, 2013.

[8] VENDRAMINI, S. H. F. et al. Tratamento supervisionado no controle da tuberculose em uma unidade de saúde de Ribeirão Preto: a percepção do doente.

Boletim de Pneumologia Sanitária, v. 10, n. 1, p. 5-12, 2002.

[9] WORLD HEALTH ORGANIZATION (WHO). Global tuberculosis control: epidemiology, strategy, financing. Geneva: WHO, 2009.

[10] WORLD HEALTH ORGANIZATION (WHO). Relevé épidémiologique hebdomadaire. Weekly epidemiological record, n. 35, p. 337-48, 2010.

MARCELA GONÇALVES graduanda em Enfermagem da Escola de Enfermagem de Ribeirão Preto (EERP-USP)

KAREN DA SILVA SANTOS mestranda da Escola de Enfermagem de Ribeirão Preto (EERP-USP) -e-mail: karen-web@hotmail.com 
FABIANA RIBEIRO SANTANA doutoranda da Escola de Enfermagem de Ribeirão Preto (EERP-USP)

CINIRA MAGALI FORTUNA professora doutora da Escola de Enfermagem de Ribeirão Preto (EERP-USP) 